\title{
Pattern recognition of motor imagery EEG using wavelet transform
}

\author{
Bao-Guo Xu \& Ai-Guo Song
}

School of Instrument Science and Engineering Southeast University, Nanjing 210096, China.

\begin{abstract}
Brain-computer interface $(\mathrm{BCl})$ provides new communication and control channels that do not depend on the brain's normal output of peripheral nerves and muscles. In this paper, we report on results of developing a single trial online motor imagery feature extraction method for $\mathrm{BCl}$. The wavelet coefficients and autoregressive parameter model was used to extract the features from the motor imagery EEG and the linear discriminant analysis based on mahalanobis distance was utilized to classify the pattern of left and right hand movement imagery. The performance was tested by the Graz dataset for $\mathrm{BCl}$ competition 2003 and the satisfactory results are obtained with an error rate as low as $10.0 \%$.
\end{abstract}

Keywords: Brain-computer interface (BCl); Motor imagery; Wavelet coefficients; Autoregressive model

\section{INTRODUCTION}

Left and right hand movement imagery can modify the neuronal activity in the primary sensorimotor areas, leading to the changes of the mu rhythm and beta rhythm. BCI requires effective online processing method to classify these EEG signals in order to construct a system enabling severely physically disabled patients to communication with their surroundings [1-4].

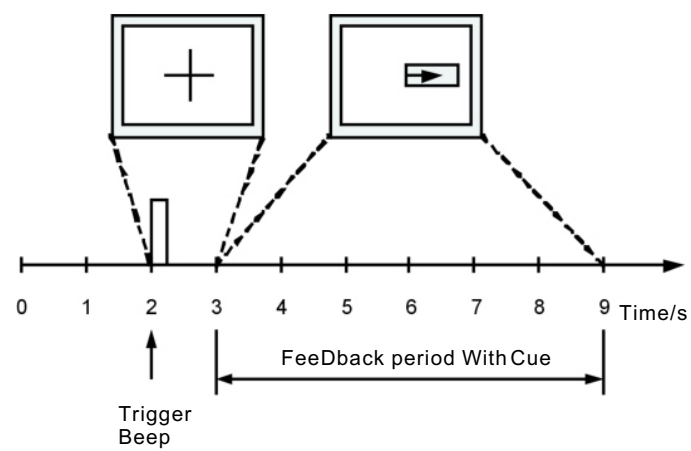

Figure1. Timing scheme.
This paper presents a novel effective method for feature extraction of motor imaginary. We combine the discrete wavelet transform (DWT) with autoregressive model (AR) to extract more useful information for non-stationary EEG signals. Applying this method to analyze the Graz dataset for BCI competition 2003, we achieved the classification accuracy of $90.0 \%$.

\section{METHODOLOGY}

\subsection{Experimental paradigm}

The data set was provided by department of medical informatics, institute for biomedical engineering, university of technology Graz [5]. It was recorded from a normal subject (female, 25y) during a feedback session. The subject sat in a relaxing chair with armrests. The task was to control a feedback bar by means of imagery left or right hand movements. The order of left and rightcues was random.

Figure 1 shows the timing of the experiment. The first $2 \mathrm{~s}$ was quite; at $\mathrm{t}=2 \mathrm{~s}$ an acoustic stimulus indicated the beginning of the trial; the trigger channel (\#4) went from low to high, and a cross "+" was displayed for $1 \mathrm{~s}$; then at $\mathrm{t}=3 \mathrm{~s}$, an arrow (left or right) was displayed as cue. At the same time the subject was asked to move a barinto the direction of the cue. The feedback was based on AAR parameters of channel \#1 (C3) and \#3 (C4), the AAR parameters were combined with a discriminant analysis into one output parameter.

The recording was made using a G.tec amplifier and a $\mathrm{Ag} / \mathrm{AgCl}$ electrodes. Three bipolar EEG chan-

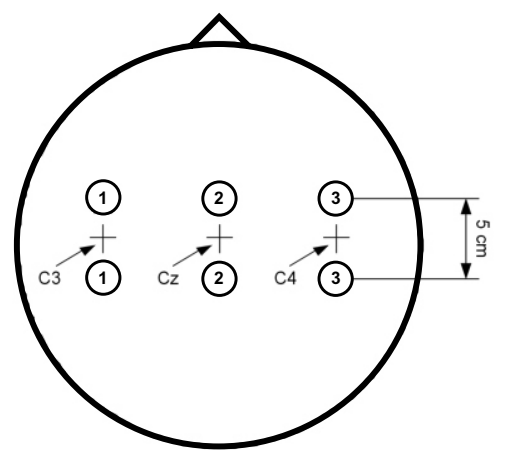

Figure 2. Electrode positions. 

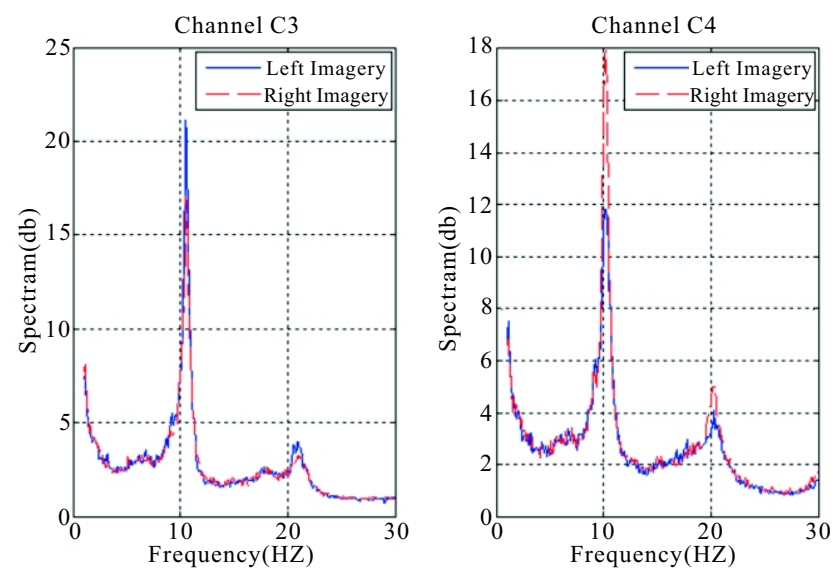

Figure 3. Average power spectrums on channelC3 and C4.

nels (anterior '+', posterior '-') were measured over $\mathrm{C} 3, \mathrm{Cz}$ and $\mathrm{C} 4$ [Figure 2]. The EEG was sampled with $128 \mathrm{~Hz}$, it was filtered between 0.5 and $30 \mathrm{~Hz}$. Similar experiments are described in [6].

The experiment consists of 7 runs with 40 trials each. All runs were conducted on the same day with several minutes break durrng experiment. One half of the datasets are provided for training; others are for evaluating the performance of the system.

\subsection{Feature consideration}

Central brain oscillations in the mu rhythm in the range of $7-12 \mathrm{~Hz}$ and beta above $13 \mathrm{~Hz}$ bands are strongly related to sensorimotor tasks. Sensory stimulation, motor behavior, mental imagery can change the functional connectivity cortex which results in an amplitude suppression or in an amplitude enhancement . This phenomenon was also called eventrelated desynchronization (ERD) and event-related synchronization (ERS) [7, 8]. Left and right hand movement imagery is typically accompanied with ERD in the mu and beta rhythms and has the characteristic of contralateral dominance.

The power spectrums on $\mathrm{C} 3$ and $\mathrm{C} 4$ of the training set are shown in Figure 3. It indicates that the power spectrums mainly distribute in the range of $8-13 \mathrm{~Hz}$ and $19-24 \mathrm{~Hz}$.In addition, the power of mu and beta rhythms evoked by right hand movement imagery is lower than that of left hand movement imagery for channel $\mathrm{C} 3$, and it is contrary for channel $\mathrm{C} 4$ which is consistent with the principle of contralateral domi- nance. This led us to use wavelet decomposition to extract the differences between the two motor imagery tasks.

\subsection{Procedure}

The flow chart of processing single-trial motor imagery EEG is shown as in Figure 4. First, the time window was used to filter the data in temporal domain in order to get the segment that contained the most obvious difference between the two motor imagery tasks. Then EEG signals were decomposed into the frequency sub-bands using DWT and a set for statistical features was extracted from the sub-bands to represent the distribution of wavelet coefficients according to the characteristics of motor imagery EEG signals. Also the sixth-order AR coefficients of segmentation EEG signals were estimated using Burg's algorithm. Next, the combination features of wavelet coefficients and the AR coefficients were used as an input vector. Finally linear discriminant analysis (LDA) based on mahalanobis distance was utilized to classify computed features into different categories that represent the left or right hand movement imagery.

\subsection{Feature extraction using discrete wavelet transforms}

Classic Fourier transform has succeeded in stationary signals processing. However, EEG signal contains non-stationary or transitory characteristics. Thus it is not suitable to directly apply Fourier transform to such signals. The wavelet transform decomposes a signal into a set of functions obtained by shifting and dilating one single function called mother wavelet $[10,11]$. Continuous wavelet transform is given by

$$
W_{f}(a, \tau)=\frac{1}{\sqrt{a}} \int_{R} f(t) \Psi^{*}\left(\frac{t-\tau}{a}\right) d t
$$

Where $\Psi(t)$ is the mother wavelet, $a$ is the scale parameter and $\tau$ is the shift parameter. In principle the CWT produced an infinite number of coefficients, thus it provides a redundant representation of the signal.

The DWT provides a highly efficient wavelet representation that can be implemented with a simple recursive filter scheme and the original signal reconstruction can be obtained by an inverse filter. The pro-

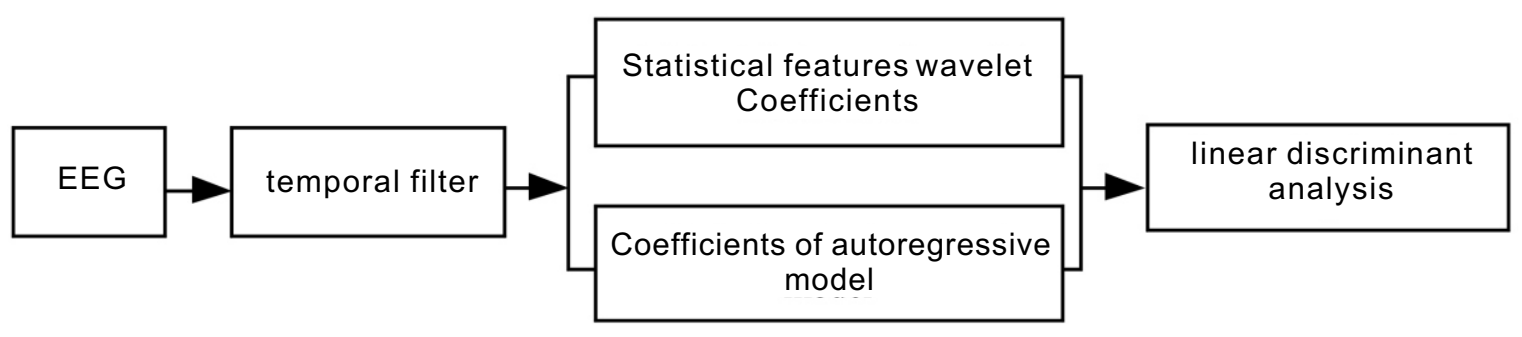

Figure 4. Flow chart of the data processing. 


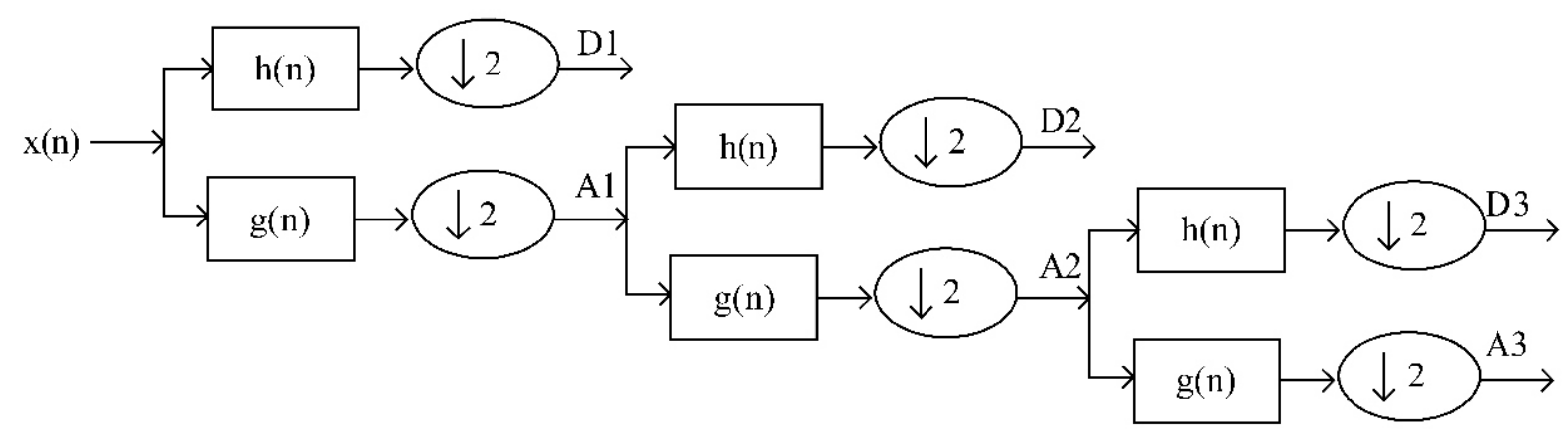

Figure 5. Decomposition of DWT; h[n] is the high-pass filter; $g[n]$ is the low-pass filter.

cedure of multi-resolution decomposition of a signal $\mathrm{x}[\mathrm{n}]$ is schematically shown in Figure 5.

The number of levels of decomposition is chosen on the basis of the dominant frequency components of the signal. According to the motor imagery EEG signals itself, we chose the level of 4 and the wavelet of Daubechies order 10.As a result, the EEG signal is decomposed into the details D1-D3 and approximation A3. The ranges of different frequency band are shown in Table 1 .

The extracted wavelet coefficients show the distribution of the motor imagery signal in time and frequency. It can be seen from the table that the component D3 decomposition is within the mu rhythm, D2 is within the beta rhythm. Statistics over the set of wavelet coefficients were computed so as to reduce the total dimension of the feature vectors. The statistical features of each sub-band are as follows:

(1) Mean of the absolute values of the coefficients.

(2) Standard deviation of the coefficients.

(3) Average power of the wavelet coefficients.

These features represent the frequency distribution and the amount of changes in frequency distribution. Thus 12 statistical features of wavelet coefficients are obtained for two channels.

\subsection{Feature extraction using autoregressive model}

EEG signal can be considered as the output of a linear filter driven by a white noise. This filter, referred to as AR, is a linear combination of the previous output itself. A zero-mean, stationary autoregressive process of order $p$ is given by

$$
x(n)=-\sum_{i=1}^{p} a_{p}(i) x(n-i)+\varepsilon(n)
$$

Where $p$ is the modelorder, $x(n)$ is the signal at the sampled point $n, a_{p}(i)$ is the AR coefficients and $\varepsilon(n)$ is a zero-mean white noise. In application, the values of the $a_{p}(i)$ have to be estimated from the finite samples of data $x(1), x(2), x(3), \ldots, x(N)$.

The first important things involved in using AR model is determining the optimal AR model order since too low a model order tends to smooth the spec- trum and too high tends to introduce spurious peaks. Here order six was used based on the suggestions [9].

Then the Burg's method was used to estimate the AR coefficients. This method is more accurate and yields better resolution without the problem of spectral 'leakage' as compared to other methods such as Levison-Durbin as it uses the data points directly. In addition, the Burg's method can minimize both forward and backward error.

Next the AR coefficients were computed and we got six coefficients for each channel, giving a total of 12 AR coefficients features for each EEG segment for a motor imagery task.

\subsection{Linear discriminant analysis (LDA)}

LDA is one of the most effective linear classification methods for brain-computer interface, and it requires fewer examples for obtaining a reliable classifier output [12].

As to the LDA method, assume that each data element $s_{i}$ has $m$ features. Then, an element $s_{i}$ is one point in a dimensional feature space. The number of examples is $n$, each example is assigned to one of two classes $C=\{0,1\}$; Then, $S$ is a matrix of size $n \times m$, and $C$ is a vector of size $n . N_{0}$. And $N_{1}$ are the number of elements for class 0 and 1 , respectively.

The mean $\mu_{\mathrm{c}}$ of each class $c$ is the meanover all $s_{i}$ with $i$ being all elements with in class $c$. The total mean of the data is

$$
\mu_{c}=\left(\frac{N_{0} \mu_{0}+N_{1} \mu_{1}}{N_{0}+N_{1}}\right)
$$

Table 1. Frequencies correspond to different levels of deposition for daubechies order 10 wavelet with a sample rate $128 \mathrm{HZ}$.

\begin{tabular}{ccc}
\hline Decomposed signal & Frequency range $(\mathrm{Hz})$ & Level \\
\hline D1 & $32-64$ & 1 \\
D2 & $16-32$ & 2 \\
D3 & $8-16$ & 3 \\
A3 & $0-8$ & 3 \\
\hline
\end{tabular}


Table 2 . Dirrerent wavelet used for extracting features.

\begin{tabular}{cc}
\hline Wavelet & Recognition rate \\
\hline Daubechies order 10 & $90 \%$ \\
Discrete Meyer & $90 \%$ \\
Coiflets order 5 & $89.29 \%$ \\
Rbio1.3 & $87.86 \%$ \\
\hline
\end{tabular}

The covariance matrix $C$ of the data is the expectation value for

$$
C=E<(s-\mu)^{T}(s-\mu)>
$$

Then, the weight vector $w$ and the offset $w_{0}$ are

$$
\begin{gathered}
w=C^{-1}\left(\mu_{1}-\mu_{0}\right)^{T} \\
w_{0}=-\mu w
\end{gathered}
$$

The weight vector $w$ determines a separating hyperplane in the $m$-dimensional feature space. The normal distance $D(x)$ of any element $x$ is

$$
D(x)=x w+w_{0}=(x-\mu) C^{-1}\left(\mu_{1}-\mu_{0}\right)^{T}
$$

If $D(x)$ is larger than $0, x$ is assigned to class 1 , while if $D(x)$ is smaller than $0, x$ is assigned to class 0 . However, $D(x)=0$ indicates that all elements $x$ are part of the separating hyperplane.

\section{EXPERIMENT RESULTS}

Here, we have had 6 statistical wavelet coefficients and $6 \mathrm{AR}$ coefficients for each channel, giving a total of 24 features for a motor imagery task. These parameters were selected as inputs of LDA classifier. Table 2 compared the classification performances among four different wavelets. The results show the Daubechies order 10 gave the best performance and the recognition rate is as high as $90.0 \%$. Also the results indicate that method of combining DWT with AR model are capable of extracting more useful information from the simultaneously acquired motor imagery EEG. Furthermore, when the window of 384 samples with a shift of 1 sample was used, maximum classification accuracy of $92.1 \%$ is achieved.

\section{CONCLUSION AND FUTURE WORK}

In this paper, a novel single-trial motor imagery EEG classification method is proposed. The pattern classification techniques as described in this work make possible the development of a fully automated motor imagery EEG signals analysis system which is accurate, simple and reliable enough to use in braincomputer interface. Future work will utilize the algorithms developed in this study to directly control the embedded rehabilitation robot so as to help the patient with severed paralysis to solve the problem of environment control and provide a new communica- tion and channel to outside world.

\section{ACKNOWLEDGEMENTS}

The work was founded by the National Basic Research Program of China (973 Program) (No.2002CB321/02), Natural Science Foundation of China (No.60475034,No.60643007) and 863 High-Tech project (No.2006AA04Z246).

\section{REFERENCE}

[1]J. Virts. The Third International Meeting on Brain-Computer Interface Technology: Making a Difference. IEEE Trans.Neural. Syst. Rehabil. Eng. 2006, 14:126-127.

[2]T. M. Vaughan. Brain-computer Interface Technology: A Review of the Second International Meeting. IEEE Trans.Neural. Syst. Rehabil. Eng. 2003, 11:94-109.

[3]J. R. Wolpaw, N. Birbaumer, and W. Heetderks, et al. Braincomputer Interface Technology: A Review of the First International Meeting. IEEE Trans. Rehabil. Eng. 2000, 8:164-173 .

[4]J. R. Wolpaw, N. Birbaumer \& D. J. McFarland, et al. Braincomputer interface for communication and control. Clinical Neurophysiology 2002, 113:767-791.

[5]B. Blankertz, K. R. Muller \& G. Curio, et al. BCI Competition 2003-Progress and Perspectives in Detection and Discrimination of EEG Single Trials. IEEE Trans. Rehabil. Eng. 2004, 51:1044-1051.

[6]A. Schlögl, C. Neuper \& G. Pfurtscheller. Estimating the mutual information of an EEG-based Brain-ComputerInterface. Biomedizinische. Technik. 2002, 47:3-8

[7]E. Houdayer, E. Labyt \& J. Cassim, et al. Relationship between event-related beta synchronization and afferent inputs: analysis of finger movement and peripheral nerve stimulations. Clinical Neurophysiology 2006, 117:628-636.

[8]G. Pfurstcheller \& F. H. Lopes da Silva. Event-related EEG/MEG synchronization and desynchronizaiton: basic principles. Clinical Neurophysiology 1999, 110:1842-1857.

[9]G. Pfurstcheller \& C. Neuper. Motor imagery and Direct BrainComputer Communication. Proc. IEEE 2001, 89:1123-1134.

[10]A. Subasi. EEG signal classification using wavelet feature extraction and a mixture of expert model. Expert System with Application, in press.

[11]A. Subasi. Automatic recognition of alertness level from EEG by using neural network and wavelet coefficients. Expert System with Application 2005, 28:701-711.

[12]A. Schlogl. A new linear classification method for an EEGbased brain-computer interface. unpublished. 